\title{
Biaxiality at the Isotropic-Nematic Interface with Planar Anchoring
}

\author{
S. M. Kamil, A. K. Bhattacharjee, R. Adhikari and Gautam I. Menon \\ The Institute of Mathematical Sciences, \\ CIT Campus, Taramani, Chennai 600 113, India
}

(Dated: December 4, 2018)

\begin{abstract}
We revisit the classic problem of the structure of the isotropic-nematic interface within GinzburgLandau-de Gennes theory, refining previous analytic treatments of biaxiality at the interface. We compare our analysis with numerical results obtained through a highly accurate spectral collocation scheme for the solution of the Landau-Ginzburg-de Gennes equations. In comparison to earlier work, we obtain improved agreement with numerics for both the uniaxial and biaxial profiles, accurate asymptotic results for the decay of biaxial order on both nematic and isotropic sides of the interface and accurate fits to data from density functional approaches to this problem.
\end{abstract}

PACS numbers: 42.70.Df,67.30.hp,61.30.Dk,61.30.Hn 
Liquid crystalline states of matter provide a useful testing ground for statistical mechanical theories of interface structure, since a variety of ordered phases can be accessed in experiments and computer simulations. The structure of the isotropic-nematic (I-N) interface presents a simple example of how interfacial order can differ radically from order in the coexisting bulk phases, since biaxial order is generically expected at the interface even if the stable ordered phase is purely uniaxial. The study of the isotropic-nematic interface was initiated in an insightful paper by de Gennes, who introduced a simple uniaxial ansatz for the tensor order parameter $Q_{\alpha \beta}$ which describes nematic order [1]. The de Gennes ansatz is exact in the absence of elastic anisotropy. However, the description of the interface in the presence of such anisotropy poses a formidable analytic and numerical problem, since the partial differential equations for the five independent components of $Q_{\alpha \beta}$ contain non-linear couplings, while $Q_{\alpha \beta}$ is itself constrained by symmetry and the requirement that its trace vanish.

Popa-Nita, Sluckin and Wheeler (PSW) [2] studied the I-N interface incorporating elastic anisotropy in the limit of planar anchoring, adapting a parametrization introduced by Sen and Sullivan[3]. In this parametrization, the principal axes of $Q_{\alpha \beta}$ remain fixed in space, and the problem reduces to the solution of two coupled non-linear partial differential equations in the dimension perpendicular to the interface. These equations represent the variation of the amplitude of uniaxial and biaxial ordering across the interface. PSW showed that the solutions of these equations exhibited biaxiality in a region about the interface [2]. The uniaxial order parameter (S) was adequately represented by a tanh profile, as in the original calculation of de Gennes, while the biaxial order parameter (T) exhibited more complex behaviour, peaking towards the isotropic side and with a trough on the nematic side. The biaxial profile was also shown to have a long tail towards the isotropic side, a feature hard to anticipate on physical grounds.

This paper extends these calculations in several new ways. First, we show that terms dropped by PSW in their simplification of the Ginzburg-Landau-de Gennes (GLdG) equations are, in fact, comparable in magnitude to the terms they retain, especially for small values of $\kappa=L_{2} / L_{1}$, the ratio of the coefficients of the two lowest-order gradient terms in the GLdG expansion. Thus, a more accurate treatment of the interface requires that these terms be retained. The resulting equations have closed form solutions in terms of hypergeometric functions. We show that such solutions provide a better description of the numerical 
data than the original calculation of PSW. We benchmark our analytic results through an accurate numerical procedure, based on a Chebyshev polynomial expansion, for the study of these equations.

We begin with the GLdG expansion of the free energy for a general $Q_{\alpha \beta}$

$$
\begin{aligned}
\mathcal{F}= & \int d z d \mathbf{x}_{\perp}\left[\frac{1}{2} A \operatorname{Tr} \mathbf{Q}^{2}+\frac{1}{3} B \operatorname{Tr} \mathbf{Q}^{3}+\frac{1}{4} C\left(\operatorname{Tr} \mathbf{Q}^{2}\right)^{2}\right. \\
& \left.+\frac{1}{2} L_{1}\left(\partial_{\alpha} Q_{\beta \gamma}\right)\left(\partial_{\alpha} Q_{\beta \gamma}\right)+\frac{1}{2} L_{2}\left(\partial_{\alpha} Q_{\alpha \gamma}\right)\left(\partial_{\beta} Q_{\beta \gamma}\right)\right] .
\end{aligned}
$$

Here $A, B$ and $C$ are expansion parameters, while $L_{1}, L_{2}$ are elastic constants. We choose $B=-0.5, C=2.67$ and $A=B^{2} / 27 C$, thus enforcing phase coexistence between an isotropic and uniaxial nematic phase [4]. The interface is taken to be flat and infinitely extended in the $x-y$ plane. The spatial variation of the order parameter only occurs along the $z$ direction $[3]$. We scale $Q_{\alpha \beta} \rightarrow Q_{\alpha \beta} / S_{c}$ where $S_{c}=-\frac{2 B}{9 C}, \mathcal{F} \rightarrow \frac{16}{9 C S_{c}^{4}} \mathcal{F}$, and measure lengths in units of $l_{c}=\sqrt{54 C\left(L_{1}+2 L_{2} / 3\right) / B^{2}}$; we choose $L_{1}=10^{-6}$ in our numerics and obtain $L_{2}$ from our choice of $\kappa$. In the case of planar anchoring, the ordering at infinity is purely uniaxial and taken to be along the $x$ axis. In this case, as shown by Sen and Sullivan, uniaxial and biaxial order vary only with $z$ and the principal axes of the $\mathbf{Q}$ tensor remain fixed in space. The form of $\mathrm{Q}$ is then

$$
\mathbf{Q}=\left(\begin{array}{ccc}
S & 0 & 0 \\
0 & \frac{1}{2}(-S+T) & 0 \\
0 & 0 & -\frac{1}{2}(S+T)
\end{array}\right)
$$

Inserting this form of $\mathbf{Q}$ into the free energy and performing the minimization yields [2]

$$
\begin{gathered}
\frac{(6+\kappa)}{(3+2 \kappa)} \partial_{z}^{2} S+\frac{\kappa}{(3+2 \kappa)} \partial_{z}^{2} T=4 S-12 S^{2}+8 S^{3}+4 T^{2}+\frac{8 S T^{2}}{3} \\
\frac{\kappa}{(3+2 \kappa)} \partial_{z}^{2} S+\frac{(2+\kappa)}{(3+2 \kappa)} \partial_{z}^{2} T=\frac{4}{3} T+8 S T+\frac{8 T^{3}}{9}+\frac{8 S^{2} T}{3}
\end{gathered}
$$

Popa-Nita, Sluckin and Wheeler now make several approximations to Eqs. 3 and 4 to solve them. First, in Eq. 3, all terms in $T$ are dropped, since $S$ is typically much larger than $T$. The resulting equation for $S$ is solved by the tanh function. In Eq. 4, PSW drop the $\frac{(2+\kappa)}{(3+2 \kappa)} \partial_{z}^{2} T$ term while retaining $\frac{\kappa}{(3+2 \kappa)} \partial_{z}^{2} S$. A test of self-consistency of this approximation is the comparison of the magnitude of these terms within the theory. Fig. 1 shows the terms 


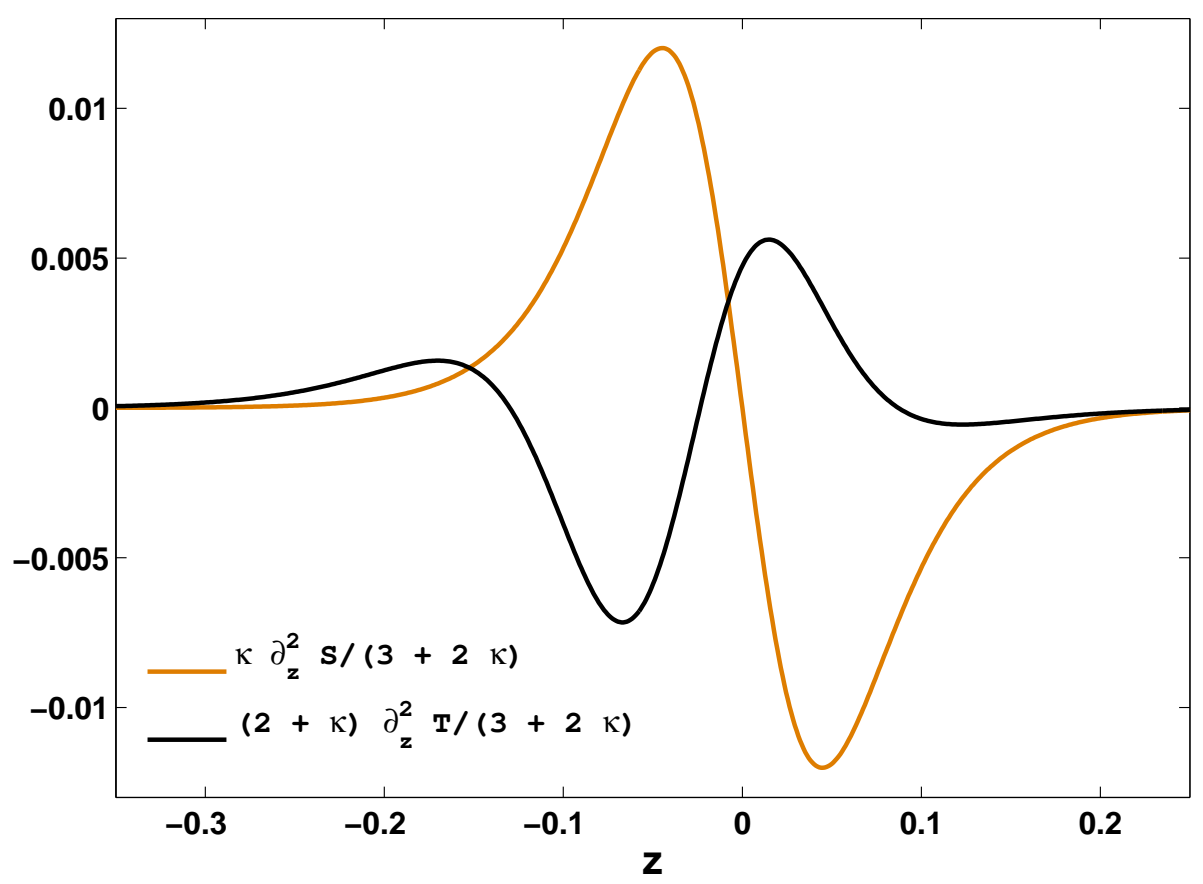

FIG. 1: (Color online) A comparison of the terms $\frac{(2+\kappa)}{(3+2 \kappa)} \partial_{z}^{2} T$ (dark line) and $\frac{\kappa}{(3+2 \kappa)} \partial_{z}^{2} S$ (light line) obtained within the PSW solution to the GLdG equations, for a $\kappa$ value of 18.0. The PSW approximation consists of ignoring the $\frac{(2+\kappa)}{(3+2 \kappa)} \partial_{z}^{2} T$ term in comparison to the $\frac{\kappa}{(3+2 \kappa)} \partial_{z}^{2} S$ term. Both terms, however, are of comparable magnitude.

$\frac{(2+\kappa)}{(3+2 \kappa)} \partial_{z}^{2} T$ (dark line) and $\frac{\kappa}{(3+2 \kappa)} \partial_{z}^{2} S$ (light line) computed through the PSW solution. As can be seen from the figure these terms only differ by a factor of order unity. Deep into the isotropic side, the term ignored by PSW exceeds the value of the term retained. Thus, while the PSW approach leads to a straightforward algebraic relation between $T$ and $S$, a more accurate method would be to retain the partial derivative term as well, requiring that we solve a partial differential equation as opposed to an algebraic one.

Our approach to this problem uses the same approximations as PSW for Eq. 3, We thus take

$$
S=\frac{S_{c}}{2}\left[1+\tanh \left(\frac{z}{\sqrt{2} \xi}\right)\right],
$$

where $\xi=\sqrt{\frac{1+\kappa / 6}{1+2 \kappa / 3}}$. Inserting this in equation (41), scaling $z$ by $\sqrt{2} \xi$, redefining the resulting quantity as $z$ again, and dropping the nonlinear term, we obtain,

$$
\partial_{z}^{2} T=2 \beta\left[\tanh ^{2}(z)+8 \tanh (z)+9\right] T+\frac{\kappa}{2+\kappa} \tanh (z)[1+\tanh (z)][1-\tanh (z)] .
$$

with $\beta=\frac{6+\kappa}{3(2+\kappa)}$. 

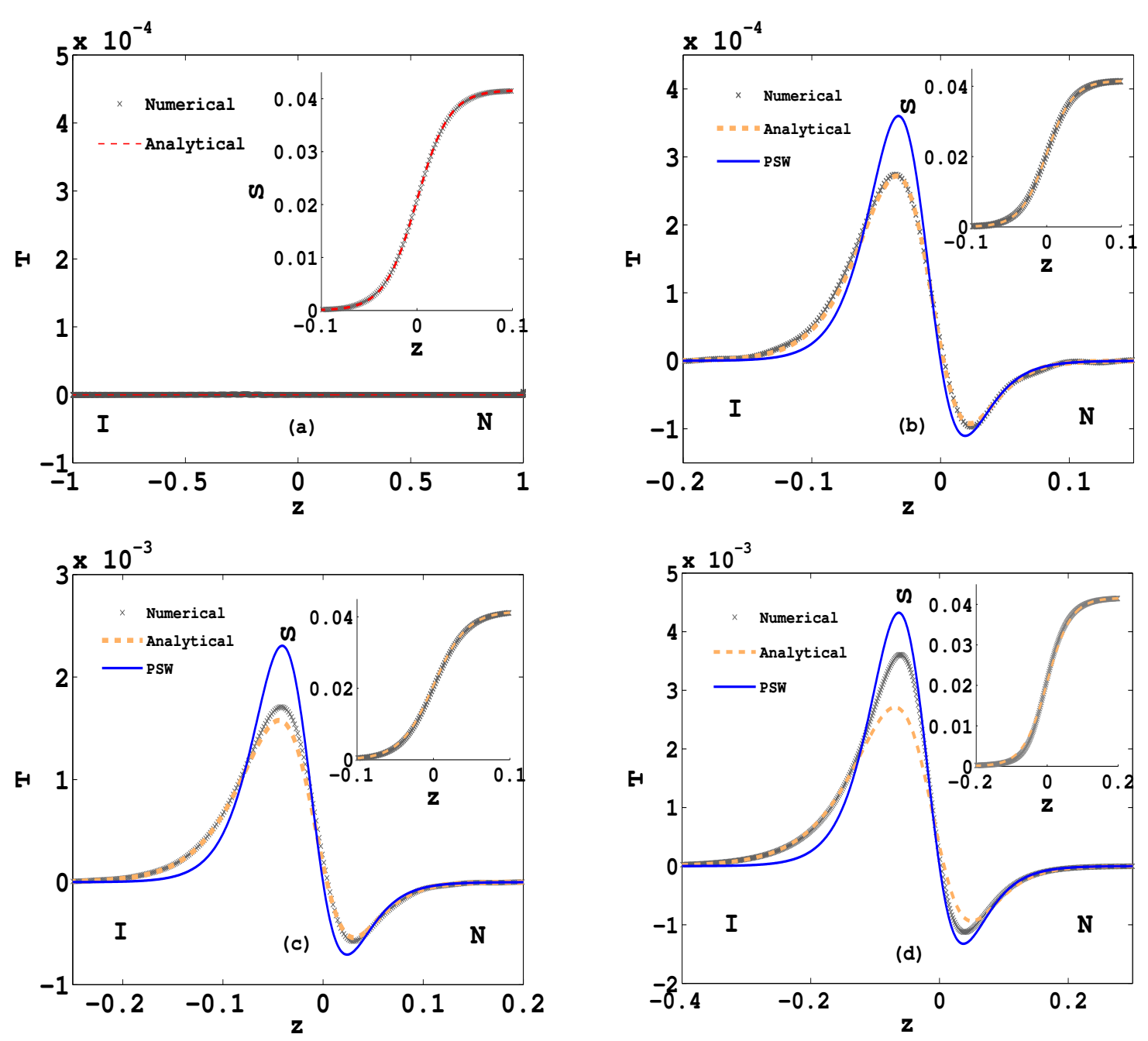

FIG. 2: (Color online) Biaxial and uniaxial profiles for $\kappa=0(a), 0.4(b), 4(c)$ and $18.0(d)$, comparing results from our numerical computations $(x)$, with our analytic formula (dashed line) and the formula of PSW (solid line). The main figure shows the biaxial profile whereas the inset shows the uniaxial profile. In (a), for $\kappa=0$, the solution has $T=0$, with the $\mathrm{S}$ profile exactly given by the tanh form. In (b), for $\kappa=0.4$, the computed biaxial profile (T) (main panel) is fit remarkably well by our analytic form, whereas the PSW approximation tends to overestimate the peak value. The uniaxial (S) profile is shown in the inset of (a); here the results obtained by us and by PSW are identical and the fit to a tanh profile is accurate over the entire region. In (c) (main panel), for $\kappa=4.0$, the numerical data are fit well by the analytic forms, particularly away from the main peak, yielding essentially exact agreement deep into the isotropic and nematic sides. The PSW approximation is still an overestimate to the peak value, and also differs sharply in relation to the numerical data deep into the isotropic side. The inset shows the uniaxial (S) profile for this case. In (d) (main panel), for $\kappa=18.0$, the PSW form appears to fit the peak better for larger $\kappa$, but again fails to capture the decay towards the isōropic side. 


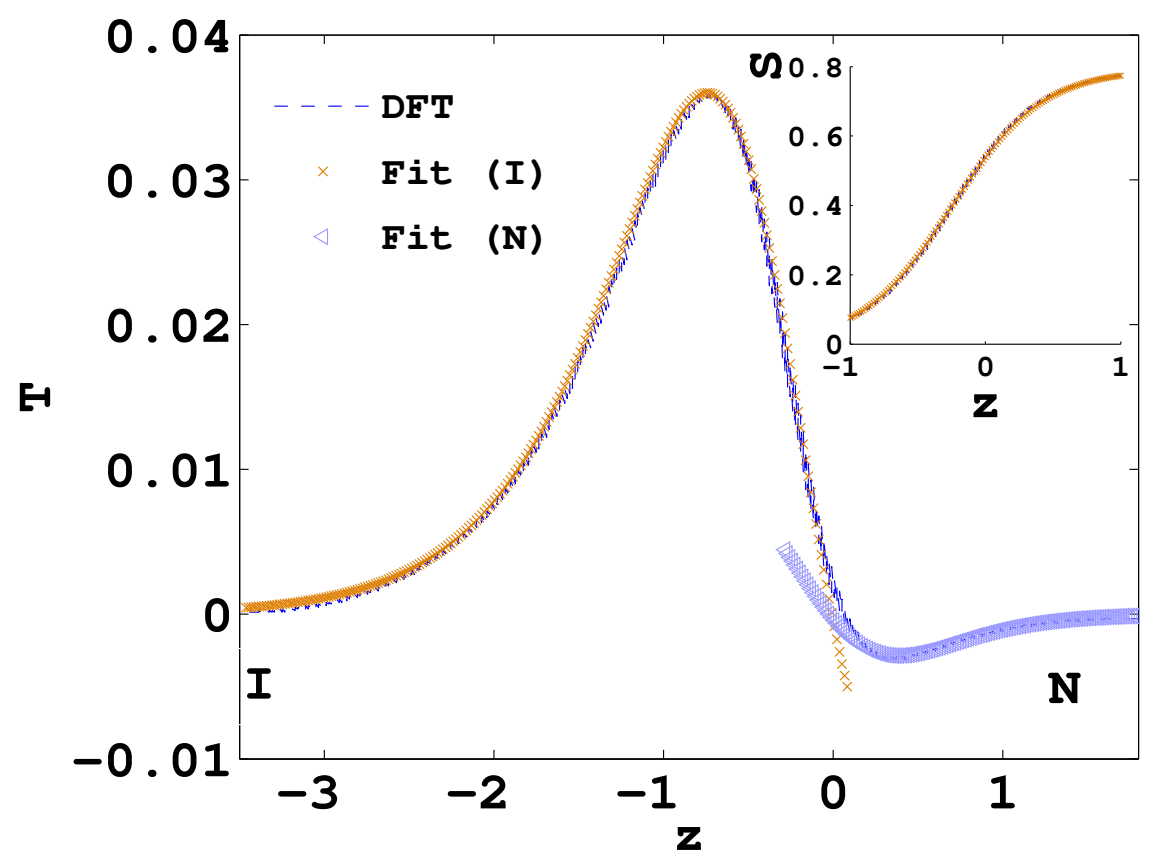

FIG. 3: (Color online) A comparison of the results of our analytic calculation to profiles of $T$ obtained from a density functional calculation for the isotropic-nematic interface. Profiles obtained for two values of $\kappa, \kappa=5.8$ (for $z<0$ ) and $\kappa=0.69$ (for $z>0$ ) are shown. The larger $\kappa$ value essentially fits the $T$ profile exactly on the isotropic side, whereas the smaller $\kappa$ value provides an accurate fit on the nematic side. The inset shows the $S$ profile obtained from the density functional calculation, together with an optimum fit varying the value of $l_{c}$

The solution of the equation consists of a homogeneous part $T_{h}$ and a particular part $T_{p}$ where $T_{h}=C_{1} t^{3 \sqrt{\beta}}(1-t)^{-\sqrt{\beta}}{ }_{2} F_{1}\left[a_{1}, b_{1}, c_{1}, t\right]+C_{2} t^{-3 \sqrt{\beta}}(1-t)^{\sqrt{\beta}}{ }_{2} F_{1}\left[a_{2}, b_{2}, c_{2}, 1-t\right]$ and $a_{1}=\frac{1}{2}+2 \sqrt{\beta}+\frac{\sqrt{1+8 \beta}}{2} ; \quad b_{1}=\frac{1}{2}+2 \sqrt{\beta}-\frac{\sqrt{1+8 \beta}}{2} ; \quad c_{1}=1+6 \sqrt{\beta} ; a_{2}=\frac{1}{2}-2 \sqrt{\beta}-\frac{\sqrt{1+8 \beta}}{2} ; \quad b_{2}=$ $\frac{1}{2}-2 \sqrt{\beta}+\frac{\sqrt{1+8 \beta}}{2} ; \quad c_{2}=1+2 \sqrt{\beta}$ and $t=\left(\frac{1-\tanh (z)}{2}\right)$. The function ${ }_{2} F_{1}$ is a hypergeometric function and $C_{1}$ and $C_{2}$ are fixed by boundary conditions.

The particular solution takes the form

$$
T_{p}(z)=\left[-y_{1}(z) I_{2}(z)+y_{2}(z) I_{1}(z)\right] / W(z),
$$

with

$$
\begin{aligned}
& y_{1}(z)=\left(\frac{1-\tanh (z)}{2}\right)^{3 \sqrt{\beta}}\left(\frac{1+\tanh (z)}{2}\right)^{-\sqrt{\beta}}{ }_{2} F_{1}\left[a_{1}, b_{1}, c_{1}, \frac{1-\tanh (z)}{2}\right] \\
& y_{2}(z)=\left(\frac{1-\tanh (z)}{2}\right)^{-3 \sqrt{\beta}}\left(\frac{1+\tanh (z)}{2}\right)^{\sqrt{\beta}}{ }_{2} F_{1}\left[a_{2}, b_{2}, c_{2}, \frac{1+\tanh (z)}{2}\right]
\end{aligned}
$$


and the Wronskian $W(z)=W=y_{1}\left(d y_{2} / d z\right)-y_{2}\left(d y_{1} / d z\right)$, where

$$
\begin{aligned}
I_{1}(z)= & \frac{2 \kappa}{2+\kappa} \sum_{m=0}^{\infty} \frac{\left(a_{1}\right)_{m}\left(b_{1}\right)_{m}}{\left(c_{1}\right)_{m} m !} t^{1+m+3 \sqrt{\beta}}(1-t)^{1-\sqrt{\beta}}\left(-\frac{2}{2+m+2 \sqrt{\beta}}\right. \\
+ & \left.\frac{m+4 \sqrt{\beta}}{(2+m+2 \sqrt{\beta})(1+m+3 \sqrt{\beta})}{ }_{2} F_{1}[1,2+m+2 \sqrt{\beta}, 2+m+3 \sqrt{\beta}, t]\right) \\
I_{2}(z)= & \frac{2 \kappa}{2+\kappa} \sum_{n=0}^{\infty} \frac{\left(a_{2}\right)_{n}\left(b_{2}\right)_{n}}{\left(c_{2}\right)_{n} n !} t_{1}^{1+n+\sqrt{\beta}}\left(1-t_{1}\right)^{1-3 \sqrt{\beta}}\left(-\frac{2}{2+n-2 \sqrt{\beta}}\right. \\
& \left.+\frac{n+4 \sqrt{\beta}}{(2+n-2 \sqrt{\beta})(1+n+\sqrt{\beta})}{ }_{2} F_{1}\left[1,2+n-2 \sqrt{\beta}, 2+n+\sqrt{\beta}, t_{1}\right]\right) .
\end{aligned}
$$

The Pochhammer symbol $(a)_{n}$ which enters above is defined via $(a)_{n}=a(a+1)(a+2) \ldots(a+$ $n-1)$. Here $t_{1}=[1+\tanh (z)] / 2$ and the result for $I_{1}(z)$ and $I_{2}(z)$ is obtained by expanding the hypergeometric functions in Eqns. 8$]$ in a power series and integrating term-by-term [5]. Note that the solutions of the homogeneous part diverge asymptotically. Thus, for the boundary condition $T=0$ at $z= \pm \infty$ the only physical solution is the particular one. Eq. 7 is thus the key analytical result of this paper, describing the variation of biaxiality across the interface. In our numerical evaluations, we sum the series for $I_{1}(z)$ and $I_{2}(z)$, retaining as many terms as are required to ensure convergence. The series in $I_{2}$ converges very fast (only 3 terms need be retained for good results) whereas the series in $I_{1}$ converges more slowly and around 9 terms must be retained for convergence. To convert these into physical units, we must undo the sequence of length transformations, replacing $z \rightarrow z /\left(\sqrt{2} \xi l_{c}\right)$.

An asymptotic analysis of these equations is possible: for $z \rightarrow-\infty, S$ and $T$ are small. The tanh profile for $S$ can be approximated as $\frac{1}{2}\left(1+\tanh \left(\frac{z}{\sqrt{2} \xi}\right)\right) \rightarrow e^{\frac{2 z}{\sqrt{2} \xi}}$ while Eq. (44) takes the form $2 \xi^{2} \partial_{z}^{2} T=4 \beta T-\left(\frac{2 \kappa}{2+\kappa}\right) e^{2 \frac{z}{\sqrt{2} \xi}}$ with $\beta=\frac{6+\kappa}{3(2+\kappa)}$. Thus $\partial_{z}^{2} T=\frac{4}{3} \frac{(3+2 \kappa)}{(2+\kappa)} T-\frac{2 \kappa(3+2 \kappa)}{(2+\kappa)(6+\kappa)} e^{2 \frac{z}{\sqrt{2} \xi}}$ with asymptotic solution

$$
T \sim e^{\sqrt{\frac{4(3+2 \kappa)}{3(2+\kappa)}} z}, z \rightarrow-\infty
$$

a result in perfect accord with the computed forms of $T$ deep into the isotropic phase. Note that $T>S$ and $(S+T) / 2>S$ as one moves deeper into the isotropic side. This implies that the principal order parameter is negative as pointed out in Ref. [2], where this result was obtained numerically. 
As $z \rightarrow \infty$, an alternative asymptotic expansion can be derived by taking $S=1-\frac{1}{2} e^{-b x}$ with $b=2 \sqrt{\frac{3+2 \kappa}{6+\kappa}}$. We then obtain

$$
T \sim e^{-2 \sqrt{\frac{3+2 \kappa}{6+\kappa}} z}, z \rightarrow \infty
$$

in agreement with our numerical results. Popa-Nita, Sluckin and Wheeler provide an analysis of the asymptotics in the specific limit that $\kappa \rightarrow \infty$. However, our results cannot be directly translated to this limit, since we assume a tanh profile of $\mathrm{S}$; this approximation becomes increasingly inaccurate for larger $\kappa$ (see below).

Our numerical results are obtained using a spectral collocation method [6], applied to our knowledge for the first time to the GLdG equations. In the spectral collocation, the solution is expanded in an orthogonal basis of Chebyshev polynomials in a bounded interval. Differentiation operators constructed from this Chebyshev interpolant are spectrally accurate, in the sense that the error vanishes exponentially in the number of retained polynomials. The interpolant is constructed so as to satisfy Dirichlet boundary conditions. Though the physical problem is for an unbounded interval, our numerical approximation of a bounded interval gives excellent results since all variation in the order parameters is restricted to the region proximate to the interface.

Specifically, we solve the equations of equilibrium

$$
\left(A+C T r Q^{2}\right) Q_{\alpha \beta}(\mathbf{x}, t)+B \overline{Q_{\alpha \beta}^{2}(\mathbf{x}, t)}=L_{1} \nabla^{2} Q_{\alpha \beta}(\mathbf{x}, t)+L_{2} \overline{\nabla_{\alpha}\left(\nabla_{\gamma} Q_{\beta \gamma}(\mathbf{x}, t)\right)}
$$

by transforming to a basis $\left\{a_{i}\right\}$ which enforces symmetry and tracelessness, as $Q_{\alpha \beta}=$

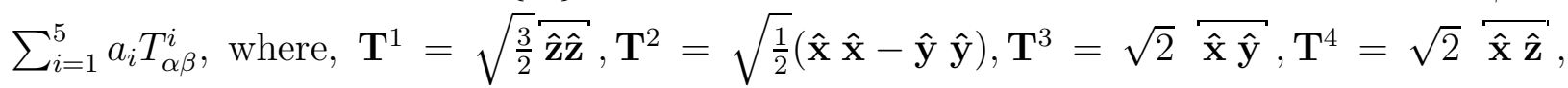
$\mathbf{T}^{5}=\sqrt{2} \hat{\hat{\mathbf{y}} \hat{\mathbf{z}}}$. Overbars indicate traceless symmetric parts. We thus obtain five simultaneous partial differential equations for the $a_{i}$, which are steady-states of the time-dependent equations we have obtained earlier [7]. Note specifically that we make no symmetry-based ansatz for the components of $Q_{\alpha \beta}[8]$.

The spectral collocation reduces these differential equations to non-linear algebraic equations. We solve them using a relaxation method from a well-chosen initial condition, relaxing till the differential change in successive iterations is less than $10^{-5}$. Spectral convergence to machine accuracy is obtained by retaining 128 Chebyshev modes, as we have checked by an explicit calculation. To compare with analytical and density functional results, the solution 
at the Chebyshev nodes is interpolated using barycentric interpolation without compromising spectral accuracy. The DMSUITE library is used for the numerical implementation [9].

Our results are summarized in Fig. 2 and Fig. 3, The main panel of Fig. 2 (a), obtained by solving Eq. 3 and 4 for a value of $\kappa=0.0$, shows the biaxiality profile obtained using our numerical spectral scheme (crosses), as compared to the analytic result of $T=0$. The uniaxiality profile shown in the inset is exactly the tanh profile obtained by de Gennes. This limit provides a simple test of our numerical methods, since the solution to Eqs. 3 and 4 in this limit is exact. Fig. 2 (b) shows the biaxiality profile obtained using our spectral scheme (crosses), as compared to the analytic results derived here (dashed line) and results obtained by PSW (solid line) for a value of $\kappa=0.4$. As can be seen, the numerical data are fit remarkably well by the analytic forms, whereas the PSW approximation tends to overestimate the peak value. The inset to Fig. 2 (b) shows the uniaxial (S) profile, obtained numerically as well as in our analytic calculation; here the results obtained by us and by PSW are identical. The fit to a tanh profile is accurate over the entire region.

The main panel of Fig. 2 (c) shows the biaxiality profile obtained using our spectral scheme (crosses), as compared to the analytic results derived here (dashed line) and results obtained by PSW (solid line) for a value of $\kappa=4$. Again the numerical data are fit well by the analytic forms, particularly away from the main peak, yielding essentially exact agreement deep into the isotropic and nematic sides. The PSW approximation is still an overestimate to the peak value, and also differs sharply in relation to the numerical data deep into the isotropic side. The inset to Fig. 2 (c) shows the uniaxial (S) profile for this case. Fig. 2 (d) shows the biaxiality profile obtained using our spectral scheme (crosses), as compared to the analytic results derived here (dashed line) and results obtained by PSW (solid line) for a value of $\kappa=18$. For these - and larger - values of $\kappa$, our analytic fits differ noticeably from the numerical data. The PSW form appears to fit better for larger $\kappa$, although we believe that this is fortuitous. It appears that the principal error arises from our approximation of the $S$ profile as a tanh form. For large $\kappa$, this approximation is less accurate.

Fig. 3 compares the results of our analytic calculation to profiles of $T$ obtained from a density functional calculation for the isotropic-nematic interface [10] a method which provides an alternative, more molecular approach to this problem[11]. We have taken numerical data for uniaxial and biaxial profiles obtained in Ref. [10], varying the free parameters $S_{c}$, 
$l_{c}$ and $\kappa$ in our solutions till an optimal fit is obtained. The values of $S_{c}$ and $l_{c}$ can be obtained from fits to $S$; thus only $\kappa$ need be varied to represent the $T$ profile. Fig. 3 shows profiles obtained for two values of $\kappa: \kappa=5.8$ (for $z<0$ ) and $\kappa=0.69$ (for $z>0$ ). The larger $\kappa$ value fits the profile very closely on the isotropic side, whereas the smaller $\kappa$ value provides an accurate fit on the nematic side [12]. It does not seem possible to fit the complete profile using a single value of $\kappa$. This could have been anticipated on physical grounds since the density functional theory yields a density difference between coexisting isotropic and nematic phases. The elastic coefficients $L_{1}$ and $L_{2}$ which enter our calculation do in principle contain a density dependence which we ignore here.

In conclusion, we have presented results for the uniaxial and biaxial profiles, in the case of planar anchoring, for the classic problem of the structure of the isotropic-nematic interface within Ginzburg-Landau-de Gennes theory. Our work refines previous analytic treatments of biaxiality at the interface. We have implemented a highly accurate spectral collocation scheme for the solution of the Landau-Ginzburg-de Gennes equations and used this numerical scheme in our tests of the analytic results.

In comparison to earlier work, we obtain improved agreement with numerics for both the uniaxial and biaxial profiles, with our results being increasingly accurate as the anisotropy is reduced. We also provide accurate asymptotic results for the decay of the $S$ and $T$ order parameters deep into the nematic and isotropic phases. Our calculated profiles show a pleasing consistency with profiles obtained from density functional approaches. Further extensions of these numerical and analytic methods to the case of an intermediate anchoring condition far from the interface are currently under way.

The authors thank C. Dasgupta for discussions. This research was supported in part by the DST(India) and the Indo-French Centre for the Promotion of Advanced Research.

[1] P. G. de Gennes, Molecular Crystals and Liquid Crystals 12, 193 (1971).

[2] V. Popa-Nita, T. J. Sluckin, and A. A. Wheeler, J. Phys. II (France) 7, 1225 (1997).

[3] A. K. Sen and D. E. Sullivan, Phys. Rev. A 35, 1391 (1987).

[4] E. F. Gramsbergen, L. Longa, and W. H. de Jeu, Physics Reports 135, 195 (1986).

[5] M. Abramowitz and I. A. Stegun, Handbook of Mathematical Functions: with Formulas, 
Graphs, and Mathematical Tables (Dover, 1964).

[6] L. N. Trefethen, Spectral Methods in Matlab (SIAM, Philadelphia, 2000).

[7] A. K. Bhattacharjee, G. I. Menon, and R. Adhikari, Phys. Rev. E 78, 026707 (2008).

[8] The ambiguity of the sign of $T$ in the uniaxial phase, or whenever the ordering of the subleading eigenvalues changes, is resolved by requiring solutions to be smooth over the computational domain.

[9] J. A. C. Weidemann and S. C. Reddy, ACM Transactions on Mathematical Software 26, 465 (2000).

[10] Z. Y. Chen, Phys. Rev. E 47, 3765 (1993).

[11] S. Wolfsheimer, C. Tanase, K. Shundyak, R. van Roij, and T. Schilling, Phys. Rev. E 73, 061703 (2006).

[12] An alternative density functional approach (Ref. [13]) yield profiles which can also be fit very well on the nematic side by our methods, although the fit towards the isotropic side is of reduced quality.

[13] K. Shundyak and R. van Roij, J. Phys.: Condens. Matter 13, 4789 (2001). 